CIUDAD Y TERRITORIO

ESTUDIOS TERRITORIALES

ISSN(P): 1133-4762; ISSN(E): 2659-3254

Vol. LII, № 206, invierno 2020

Págs. $861-874$

https://doi.org/10.37230/CyTET.2020.206.09

CC BY-NC 4.0

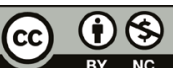

\title{
Exclusión laboral por movilidad. El caso de Quito (Ecuador)
}

\section{Susana Herrero-Olarte}

Coordinadora Centro de Investigaciones Económicas y Empresariales, Universidad de Las Américas (Quito, Ecuador).

RESUMEN: En esta investigación se analiza si en la ciudad de Quito la movilidad es un factor condicionante del acceso al empleo, lo que estaría condicionando los niveles de desarrollo alcanzados. Los resultados del modelo de regresión por medio de Mínimos Cuadrados Ordinarios aplicado muestran que la movilidad es un factor de exclusión laboral al relacionarse inversamente en el territorio con la pobreza multidimensional. Además, el lugar de residencia condiciona las oportunidades y perpetúa las estructuras que limitan el desarrollo. Específicamente, los resultados sugieren que la distancia hasta la principal fuente de ingresos medida a través del tiempo y la densidad de los medios de transporte en cada parroquia fomentan la generación de pobreza.

PALABRAS CLAVE: Desarrollo urbano; Exclusión laboral; Movilidad; Acceso al empleo; Quito.

\section{Labor exclusion due to mobility. The case of Quito (Ecuador)}

ABSTRACT: This research analyses whether in the city of Quito mobility is a determining factor in access to employment, which would be conditioning the levels of development achieved. The results of the regression model through Ordinary Least Squares show that mobility is a factor of labor exclusion as it is inversely related to multidimensional poverty in the territory. In addition, the place of residence conditions the opportunities and perpetuates the structures that limit development. Specifically, the results suggest that the distance to the main source of income measured over time and the density of the means of transportation in each parish foster the generation of poverty.

KEYWORDS: Urban development; Labor exclusion; Mobility; Access to employment; Quito.

Recibido: 06.09.2019; Revisado: 04.03.2020

Correo electrónico: susana.herrero@udla.edu.ec; No ORCID: https://orcid.org/0000-0003-3509-6316

La autora agradece los comentarios y sugerencias realizados por los evaluadores anónimos, que han contribuido a mejorar y enriquecer el manuscrito original. 


\section{Introducción}

a pobreza pasó de medirse a través de los ingresos para cubrir las necesidades a relacionarse a mediados del siglo XX directamente con las necesidades no cubiertas, tomando un carácter cualitativo o multidimensional. Desde el concepto de exclusión, para satisfacer las necesidades es necesario tener acceso a las distintas formas de cubrirlas. La pobreza responde por lo tanto a la falta de oportunidades.

Cuando no es posible o es muy difícil llegar físicamente hasta las oportunidades para cubrir las necesidades, nos referimos a la exclusión por movilidad. Están por lo tanto en condición de exclusión por movilidad las personas que no pueden o les es muy difícil llegar a las herramientas que les permitan superar su condición de pobreza.

Entre las herramientas que pueden utilizarse para superar la condición de pobreza el empleo cobra especial importancia no sólo por su efecto directo sobre la exclusión económica, sino también sobre la política y la cultural.

En este trabajo se profundiza en el concepto de exclusión por movilidad estudiando la dificultad física para acceder al empleo en función del lugar de residencia como límite para superar la pobreza. El análisis se realiza en la ciudad de Quito con el objetivo de acercar en la práctica el concepto de exclusión en Sudamérica, y atendiendo a que se espera que las ciudades de más de un millón de habitantes, sin ser megalópolis, sean las que más crezcan en la región.

La hipótesis que se pretende contrastar es que la menor movilidad desde la parroquia de residencia a la parroquia que más empleos genera, condiciona la tasa de pobreza de la parroquia de residencia.

Para el análisis se ha aplicado un modelo de correlación en el que la variable dependiente es el Índice de Pobreza Multidimensional (IPM)

\footnotetext{
1 Los primeros análisis vienen de la mano de Adam Smith (Cfr. Heilbroner, 1976), David Ricardo (Cfr. Sraffa, 1986) o Malthus (1846). Los primeros análisis cuantitativos son los de Bоотн (1889) y Seebohm, en 1908 (Cfr. RobeRTs, 1977). ${ }^{2}$ Entre los primeros estudios desde la economía del desarrollo que buscaban la causalidad entre la pobreza y los ingresos, equivalentes a variables macro económicas como el Producto Interno Bruto (PIB) per cápita, destacan los trabajos de Rosenstein-Rodan (1943), Prebisch (1950), NuRkse, (1953), SCITOVSKY, (1954), SWAMY (1967), SHASHUA \& GoldSCHMIDT (1972) y Demery \& Demery (1973).
}

y las independientes las variables de movilidad. El cálculo del IPM por parroquias para Quito se ha realizado en base a la metodología utilizada a nivel nacional para el cálculo del Índice por cantones, basada a su vez en la del Oxford Poverty \& Human Development Initiative (OPHI) de ALKIRE \& Foster (2007). Los indicadores de movilidad utilizados han sido la distancia en tiempo entre cada parroquia y la parroquia que más empleos genera, la parroquia de Iñaquito, y la densidad de medios de transporte, calculando la cantidad de líneas y la frecuencia de paso. Como variable de control se incluye la etnia.

Las variables del modelo resultan significativas, lo que muestra cómo la distancia en tiempo hasta la parroquia que más empleos genera y la densidad de los medios de transporte, es decir, las variables de movilidad seleccionadas, condicionan los niveles de pobreza.

\section{Mejorar las oportunidades para superar la pobreza}

Los primeros estudios teóricos y análisis empíricos económicos relacionaban la pobreza con unos ingresos insuficientes para alcanzar niveles mínimos de subsistencia ${ }^{1}$. Para este enfoque cuantitativo o unidimensional de la pobreza, las personas están en condición de pobreza cuando no cuentan con los ingresos suficientes para pagar los bienes y servicios básicos. La causa de la pobreza es una, la falta de dinero. La forma de reducir la pobreza es, por lo tanto, aumentar los ingresos o mejorar los indicadores económicos equivalentes incidiendo en las variables macroeconómicas. Este tratamiento de la pobreza es la base de la economía del desarrollo².

Durante la década de los cuarenta, desde las distintas ciencias sociales se realizó casi simultáneamente una revisión de la idea que se tenía de las necesidades humanas, generando una nueva 
categorización ${ }^{3}$. Las distintas necesidades que pue-de tener una persona, que pueden cambiar en el tiempo y el espacio, llevaron a cuestionar si, como hasta entonces, el dinero era la única forma de cubrirlas. Para tratar la pobreza, era entonces necesario relacionarla directamente con las necesidades que no estaban satisfechas y no con el dinero que permitiera comprar los bienes y servicios para cubrirlas. Surge así un nuevo planteamiento de la pobreza, cualitativo o multidimensional, que iba más allá de los ingresos para su valoración.

El enfoque cualitativo o multidimensional de la pobreza necesitaba generar una nueva forma de medir la pobreza. Hasta entonces había sido sencillo porque según el enfoque cuantitativo o unidimensional, los ingresos permitían conocer si una persona estaba o no en condición de pobreza. En 1954 las Naciones Unidas (NNUU) crearon un comité para crear un índice capaz de medir las condiciones de vida comparables entre países que fueran más allá de los ingresos. El índice, que se propuso teóricamente, nunca llegó a calcularse. El primer índice que sí llegó a calcularse fue el desarrollado por DREWNOWSKI \& ScOTT (1966), que desde el United Nations Research Institute for Social Development (UNRISD) presentaron un primer indicador para medir el estándar de vida para veinte países, que incluía variables de educación, salud, nutrición, habitabilidad, ocio, seguridad y capacidad de ahorro. El problema de este primer indicador era poder calcularlo en los países de renta media y baja, donde los datos eran escasos, pero donde más importante era poder hacerlo dado los volúmenes de pobreza que registraban, aún en una valoración cualitativa. El esfuerzo desde las distintas agencias para el desarrollo de las NNUU por generar un índice para calcular la pobreza cualitativa o multidimensional ponía de manifiesto su compromiso con este nuevo enfoque. Seers (1969), el primer director del inglés Institute of Development Studies (IDS), sumaba esfuerzos con las NNUU comprometiendo al recién nacido IDS con el enfoque cualitativo y multidimensional de la pobreza.

Al compromiso desde las agencias de desarrollo de las NNUU y el IDS se sumó el del Banco Mundial, especialmente importante por peso en cuestiones de desarrollo ante el resto de actores. Seers presentó a su colega, el economista paquistaní Mahumat at Haq, a Robert McNamara (PEARSON, 1969), recientemente nombrado director del Banco Mundial (JoLly, 2008). Mahumat at Haq comulgaba activamente con una visión de la pobreza cualitativa y multidimensional. En 1970 , McNamara nombraba a Haq asesor económico principal y responsable de la política del Banco Mundial. Relacionado académicamente con el Nobel hindú de economía Amartya Sen (SEN, 2008), a quien haré referencia en adelante, Haz continuaba, junto a su colega Sen, los recientes avances del resto de las ciencias sociales y de la historia de la India y Paquistán (DESAl, 2001), donde el análisis y la valoración de la pobreza y las necesidades y una nueva concepción del desarrollo basado en las oportunidades habían impulsado recientes cambios políticos en su país.

Haq, ya desde el Banco Mundial, declaraba en 1971 que equiparar el Producto Nacional Bruto (PNB) per cápita a la pobreza era un error y que la pobreza era una cuestión de oportunidades (JAMESON, 1980), posición que respaldaba públicamente el propio McNAmARA (1972). En el primer Informe sobre Desarrollo Mundial del Banco Mundial, de 1978, la pobreza se presentaba como una cuestión multidimensional que debía medirse en función de las necesidades no cubiertas, y no considerando exclusivamente los ingresos (MAWDSLey \& RigG, 2003).

La proliferación de los posicionamientos en defensa de la consideración multidimensional de la pobreza cobraba importancia al publicarse cada vez más estudios que cuestionaban la fiabilidad del enfoque cuantitativo o unidimensional de la pobreza para poder saber si una familia tenía cubiertas sus necesidades básicas ${ }^{4}$.

El consenso sobre la necesidad de considerar la pobreza como una cuestión cualitativa o multidimensional invitaba a desarrollar un indicador capaz de medirla en distintos países, incluyendo especialmente a los países de ingreso medio y bajo.

Surgieron entonces nuevos índices de referencia para medir la pobreza cualitativa o multidimensional,

\footnotetext{
${ }^{4}$ Morawetz (1977) relacionaba débilmente al PIB per cápita con las necesidades básicas, cuantificando el vínculo con un R2 de 0,11 (MorAWETZ, 1979). Hicks \& STREETEN (1979), economistas del Banco Mundial, mostraban cómo el aumento en la esperanza de vida llegaba a declinar al alcanzar cierto nivel de ingresos. Hicks (1979), si bien relacionaba el
} 
como el indicador de las Necesidades Básicas Insatisfechas (NBI) de la Comisión Económica para América Latina (CEPAL), propuesto en 1989, el Índice de Pobreza Humana (IPH) y el IPH-2 de las NNUU, en 1997 y 1998, y el IPM, presentado en 2010 por las NNUU y el OPHI de la Universidad de Oxford (ALKIRE \& FOSTER, 2007). EI IPM atiende a las necesidades de educación, trabajo y seguridad social, salud, agua y alimentación y hábitat, vivienda y ambiente sano. En el Anexo 1 se recoge el detalle de cada variable incluida.

Dado que las necesidades humanas pueden satisfacerse de distintas formas, la pobreza cualitativa o multidimensional podrá por lo tanto reducirse también de varias maneras. Esta es la premisa de la que parte Sen para desarrollar las propuestas que le harán ganar el premio Nobel de economía.

Sen, influido desde la sociología y la psicología, consideraba que era fundamental que los individuos pudieran acceder a los bienes y servicios, es decir, que tuvieran la titularidad o el derecho sobre ellos. El acceso a los bienes podía darse de tres formas: comprando los bienes, produciendo los bienes o recibiéndolos a modo de donación. Una vez la persona contaba con la titularidad sobre los bienes y servicios para cubrir sus necesidades era necesario saber cómo utilizarlos para poder satisfacerlas. Era lo que SEN $(1981,1982,1984)$ llamaba tener el funcionamiento. Para DRĖZE \& SEN (1991) una persona era pobre cuando no tenía la titularidad sobre los bienes y servicios que le permitía cubrir sus necesidades para ejercer y desarrollar su vida en libertad y con todo su potencial si así lo quisiera; o cuando tenía la titularidad, pero no tenía el funcionamiento. Para SEN (1999), tener la titularidad y el funcionamiento sobre los bienes y servicios para cubrir sus necesidades se traducía en tener las oportunidades para que una persona tuviera la capacidad de ser libre. Las oportunidades se convirtieron, tal y como ya habían anticipado BAUER (1957) y STREETEN (1984), en la cuestión central e indiscutible para el análisis de la pobreza en adelante.

Ante las voces críticas que consideraban que las oportunidades eran una cuestión subjetiva que dependían de la propia percepción del individuo, no podía obviarse el hecho de que existiesen ciertos parámetros objetivos generalmente aceptados de oportunidades fundamentales para poder dar cobertura de las necesidades básicas (VON Hoegen \& Palma, 2000).

El límite a las oportunidades se ha desarrollado especialmente desde el concepto de exclusión. El concepto de exclusión surgió con fuerza en Francia de la mano de LENOIR (1974), refiriéndose a la falta de oportunidades como causa de la pobreza. En la década de los ochenta la idea de exclusión permitía en Europa referirse a las personas sin oportunidades de mejora a las que el Estado no podía atender por los cambios estructurales y sistémicos nacionales del momento fruto del debilitamiento del sistema de protección social y de la flexibilización del mercado de trabajo (ARRIBA, 2002).

Desde los Estados Unidos (EEUU) y en un contexto similar, HARRINGTON (1984) aplicaba el concepto de "nueva pobreza" y Wilson (1987) de "infraclase urbana" para referirse, al igual que en el caso de exclusión, a la situación en la que se encontraban los que padecían las desventajas en los ámbitos de la salud, la educación, el empleo, la vivienda, etc. y que no tenían acceso a las oportunidades para superarlas, como señalaba ABRAHAMSON (1997).

La Comisión de las Comunidades Europeas contribuyó sustancialmente al desarrollo teórico del concepto de exclusión ${ }^{5}$. Junto a los EEUU, la CE desarrolló varias estrategias nacionales y regionales para mejorar las oportunidades de los que no las tenían en las dimensiones económica, política y cultural. La exclusión económica, en referencia a la falta de acceso a fuentes de empleo y al mercado de bienes y servicios; política, asimilable a la falta de acceso a derechos civiles y políticos; y cultural, equiparable a la falta de similitud étnica, de género, generacional, religiosa, etc. con el promedio o el ideal del grupo general.

Pese al avance realizado, todavía el concepto de exclusión presenta desafíos sustanciales. No se ha logrado superar la dificultad que, en la práctica, supone incorporar en una sola palabra procesos de exclusión que "[s]e desarrollan en diversos ámbitos y desembocan en situaciones de naturaleza vari[a]» (COMISIÓN DE LAS COMUNIDADES EUROPEAS, 1992, p. 31), y que lidian con privaciones que en lo cotidiano pueden ser en parte subjetivas y cambiantes (SILVER, 1994). No se ha alcanzado el consenso sobre la forma de medir y valorar la condición de exclusión, temiéndose incluso que no se alcance nunca (RAYA, 2005). Tampoco el concepto en sí está claro. Suele equipararse, en yuxtaposición al de pobreza cuantitativa, al de pobreza multidimensional (DELEECK \& VAN DEN BOSCH, 1992 Y TEZANos, 1999), lo que cuestiona su uso teórico y minimiza su importancia práctica. Incluso en el análisis comparativo, la medición de la exclusión se apoya en indicadores propios de la pobreza cuantitativa (ARRIBA, 2002). Surge además la duda sobre las posibilidades de éxito al 
intentar adaptar el concepto de exclusión que nace y se desarrolla en Europa y EEUU, fruto de un cambio estructural, a los países de ingresos bajos, que mantienen unas condiciones en las que los excluidos son en realidad una mayoría.

Pese a los retos que supone la aplicación del concepto de exclusión, la idea sigue siendo prometedora. Es la plataforma para un desarrollo más justo y equitativo que iguale un mínimo de oportunidades para que las personas puedan escoger su camino y protagonizar su desarrollo, lo que las hará libres y liberará además a la sociedad de parte de la responsabilidad sobre su estado y futuro. En la práctica, el trabajo en torno a la exclusión posibilita identificar las causas de la falta de oportunidades que tienen en común los individuos que forman el grupo desfavorecido (KABEER, 2010), lo que facilita el desarrollo de políticas orientadas por resultados. Al incidir en las causas en lugar de incidir en las consecuencias permite acuerdos de mínimos entre las partes de distinta ideología (DE HAAN, 1999). Permite además dirigirse a los grupos especialmente vulnerables, los que dado que originalmente no tenían más en común que la misma falta de oportunidades, son más heterogéneos y a su vez los menos proclives a colaborar entre sí (AlEsINA \& al., 1999; MıgUEL \& GugERTY, 2005).

\section{El acceso físico reducido al empleo como causa de exclusión}

Entre las oportunidades para poder salir de la pobreza destaca el empleo. El acceso al trabajo mejora los componentes de la pobreza multidimensional independientemente de su naturaleza. Incide además no sólo en la exclusión económica; sino que se considera la principal vía para erradicar la exclusión política y cultural (SAITH, 2001; Barriga, 2005; Leal Maldonado \& Domínguez Pérez, 2008).

El acceso al empleo puede verse limitado por varias cuestiones, como son el nivel educativo, la apariencia o los contactos en redes. En este trabajo nos concentraremos en la relación entre el lugar de residencia y el lugar de trabajo. Vivir lejos de los puestos de empleo y no contar con medios de transporte para desplazarse puede limitar el acceso a una de las principales formas para superar la pobreza como es el trabajo.

El estudio de la accesibilidad o la forma en que las personas acceden en costo, tiempo y facilidad razonable (SEN, 2003) a su espacio de actividades, entendiendo como tal el que utilizan para desarrollar sus actividades (Golledge \& STIMSON, 1997 ) viene desarrollándose desde el estudio de la movilidad, entendida como el desplazamiento considerando los parámetros espacio y tiempo (MAY \& THRIFT, 2001; FERIA TORIBIO, 2010). Los estudios sobre la movilidad desde la sociología, la ecología, el urbanismo o la geografía han generado patrones para entenderla (BECKMANN \& al., 1983; SCHÖNFELDER \& AXHAUSEN, 2002) y llaman la atención sobre la necesidad de mejorar los sistemas de transporte y de acceso ( $\mathrm{CHURCH} \&$ al., 2000) y diversificar las opciones de la ciudadanía para movilizarse (CASS \& al., 2005).

En esta investigación se profundiza en el límite al acceso al empleo como un posible elemento de exclusión. El límite se analiza como el resultado de una movilidad insuficiente desde el barrio de residencia, hasta el lugar de trabajo.

La investigación se desarrolla en la ciudad de Quito con el objetivo de acercar la aplicación práctica del concepto de exclusión en los países de renta media y por ser una ciudad sudamericana de más de un millón de habitantes, sin ser una megalópolis, el tipo de ciudad que se espera aumente más su población en el futuro. Hasta 2030 , se espera que la población rural se estabilice, e incluso que descienda, mientras que urbana aumenta en un 18\%, llegando a los 380 millones (FIG. 1). Con respecto al aumento esperado de la población en el ámbito urbano se tiene que las ciudades de menos de un millón de habitantes se espera que tiendan a decrecer su población, las ciudades más pobladas de cada país se espera que tiendan a crecer, si bien el crecimiento es mayor en el caso de las ciudades de más de un millón de habitantes pero que no son las más pobladas (FIG. 2).

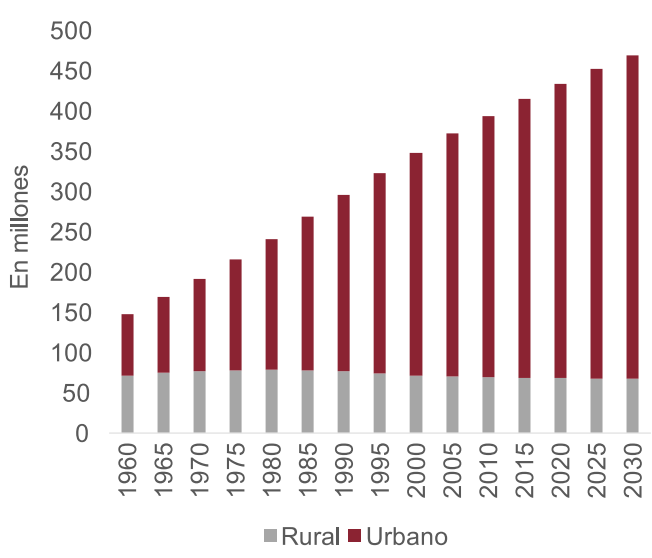

FIG. 1/ Población rural y urbana en Sudamérica (1960-2030).

Fuente: NNUU, 2014 


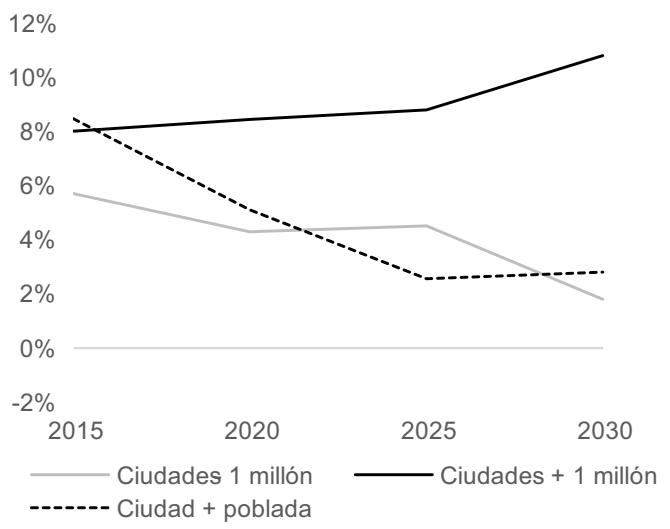

FIG. 2/ Variación respecto a los quinenios anteriores de la población urbana en Sudamérica $\left(^{*}\right)$ Los países considerados son Argentina, Brasil, Bolivia, Chile, Colombia, Ecuador, Paraguay, Perú y Uruguay.

Fuente: NNUU, 2014

\section{La movilidad en Quito}

Quito, capital del Ecuador, tiene casi tres millones de habitantes y está a casi 3.000 metros de altura. La forma de la ciudad de Quito, como en casi todas las capitales latinoamericanas, responde a las necesidades de las clases dominantes. Las clases dominantes creaban barrios que abandonaban décadas después porque dejaban de responder a sus necesidades de espacio, naturaleza y exclusividad. Creaban nuevos lugares de residencia, definiendo así el patrón de la ciudad, ante unas autoridades muy limitadas en términos de intervención urbanística. Los espacios entre los barrios que iba creando la clase dominante se iban colonizando o quedaban sin ocupar dando lugar a una ciudad dispersa, que en las últimas décadas ha superado su límite natural como valle para sumar nuevos territorios de los pueblos aledaños (Bustamante-Patiño \& HerRero-Olarte, 2017).

El aumento de la delincuencia afianzó en los noventa la idea de la urbanización alejada y apartada del resto de la ciudadanía para protegerse. El comercio formal, regido por las clases dominantes, ha pasado de calles vivas comerciales a ubicarse en grandes superficies cerradas. Estos dos factores, junto a un limitado transporte públi$\mathrm{co}$, ha impulsado la adquisición de vehículos. Se reduce el interés para caminar en la ciudad, que se vive desde el coche, limitando la generación de negocios y empresas formales a pie de calle, y encareciendo el suministro de servicios en la ciudad. Los esfuerzos desde el urbanismo se dirigen a mejorar la movilidad desde el coche (PARRAdo-Rodríguez, 2018).
La dispersión de la ciudad y su predisposición al coche producen una ciudad con múltiples centralidades que no han logrado realizarse en todo su potencial. Pese al policentrismo de Quito, todavía la dependencia hacia la centralidad de la parroquia de Iñaquito es indiscutible. La parroquia de Iñaquito arranca su liderazgo en la ciudad de Quito a raíz del descubrimiento de Petróleo en el Ecuador, que llevó a un crecimiento promedio anual del 7,4\% entre 1974 y hasta 1982 (BANCO MundIAL, 2019). Con los terrenos recientemente recalificados para urbanizarse, la parroquia de Iñaquito surgió como un espacio residencial de la clase dominante, con varios centros comerciales; también como centro financiero y de gobierno al albergar varias sedes bancarias y ministerios (CARRIÓN \& al., 1987). Los nuevos barrios que crearon después por la clase dominante no lograron desplazar la hegemonía de la parroquia de Iñaquito como centro económico de la ciudad (DURÁN \& al., 2016).

Actualmente, la parroquia de Iñaquito es la que genera el mayor número de empleos, el $22,5 \%$ de toda la ciudad, según el Municipio de Quito. Se caracteriza además por presentar los mayores niveles de cumplimiento de la legislación laboral, en un contexto económico caracterizado por una mayoría de pequeñas y nano empresas concentradas en pocas ramas económicas con un freno estructural para capitalizarse y que realizan un limitado esfuerzo para mejorar su capital humano (Herrero-Olarte \& al., 2019).

El norte y el sur de la ciudad, en los extremos, alberga los grupos en peor condición de exclusión. Son extensiones urbanas que se forman por los migrantes que llegan desde el campo, que nacen como asentamientos, y que cobran legitimidad después, junto a los servicios básicos (CARRIÓN, 1989).

La hipótesis en esta investigación es que el barrio en el que una persona reside limita el acceso al empleo y genera mayores niveles de pobreza. La investigación se desarrolla en la ciudad de Quito.

\section{Metodología}

La mayoría de estudios se han desarrollado en los países de ingresos altos y estiman que una menor movilidad para llegar a las principales fuentes de ingresos implica más pobreza. Este trabajo pretende valorar si efectivamente la relación descrita se cumple en la ciudad de Quito, es decir, si tener menor movilidad para acceder al empleo según la parroquia de residencia aumenta la pobreza. 
Para realizar el análisis se va a aplicar un modelo econométrico de Mínimos Cuadrados Ordinarios (MCO). La variable dependiente es la pobreza calculada para cada una de las parroquias urbanas de la ciudad de Quito. Las dos variables independientes relacionadas con la movilidad son la (a_distanciaentiempolñaquito), que es el tiempo en minutos que toma llegar desde cada parroquia hasta la parroquia que genera más empleos, que es la parroquia central de Iñaquito ${ }^{6}$, y la (b densidadtp), que mide la disponibilidad de medios de transporte de cada parroquia. Además, se incluye, a modo de variable de control independiente, la variable (etnia).

Entre las variables tradicionalmente utilizadas para medir la movilidad destacan el tiempo para llegar, el coste del transporte, las oportunidades u opciones disponibles y su utilidad. Las aquí seleccionadas, la distancia en tiempo y la disponibilidad de medios de transporte públicos, responden al uso tradicional en ciudades de tamaño similar a la ciudad de Quito, tal y como recogen con detalle BarAdaran \& RAMJeRdi (2001) y SHOVe (2002).

En tanto a la variable de control, suele utilizarse la etnia por la tradicional relación de los grupos minoritarios como son los indígenas o los afroamericanos con la pobreza en Sudamérica en general y el Ecuador en particular.

El resultado mostrará si la ubicación de la parroquia de residencia y la disponibilidad de medios de transporte aumentan la probabilidad de ser pobre. En otras investigaciones que se desarrollen en otras ciudades podría considerarse el coste del transporte. En el caso de Quito es irrelevante porque el transporte público está integrado, es decir, cuesta 25 centavos independientemente del trayecto o de la cantidad de vehículos que haya que tomarse. EI MCO aplicado sigue la Fórmula (1):

(1) $I P M_{1}=\beta_{0}+\beta_{1}$ a_distanciaentiempolñaquito $+\beta_{2} b_{-}$densidadtp $+\beta_{3}$ c_etnia $a_{i}+u$

Donde (IPM) es el Índice Multidimensional de la Pobreza, la (distanciaentiempolñaquito) es el tiempo para llegar al trabajo, la (densidadtp) es la densidad de los medios de transporte público, (etnia) es la cantidad de indígenas por parroquias e (i) es cada una de las parroquias.

Para calcular la pobreza multidimensional a nivel de parroquia en el cantón Quito se utilizó la metodología propuesta por el Instituto Nacional de Estadísticas y Censos (INEC) para el cálculo del IPM, elaborado a partir del trabajo de Alkire \& Foster (2007). Para el análisis de la pobreza de las parroquias de Quito se utiliza una base de datos distinta que el INEC. EI INEC utiliza la Encuesta Nacional de Empleo, Desempleo y Subempleo (ENEMDU) de 2014. Como la base no permite descender más allá del cantón porque se pierde representatividad ${ }^{7}$, ha sido necesario acudir al último Censo de Población y Vivienda de 2010 (INEC, 2011) $^{8}$. De los diez indicadores que pueden calcularse utilizando la ENEMDU pueden calcularse con el Censo de Población y Vivienda (2010) ocho, lo que no resulta perjudicial en la medida en que se compararán entre sí los datos parroquiales, calculados de la misma manera. En la FIG. 3 se recogen los indicadores utilizados en el presente trabajo con el Censo, así como las ponderaciones, y los utilizados para el análisis nacional del INEC con la ENEMDU.EI IPM se calcula multiplicando la Tasa de Pobreza Multidimensional (TPM) por la Intensidad de la Pobreza (IP). La TPM se calcula como el porcentaje de la ciudadanía que cumple con tres de los ocho indicadores de pobreza. Si alcanza la mitad de los indicadores sería un pobre extremo. La IP se calcula ponderando los indicadores, dándole más importancia a unas privaciones que a otras. Los detalles de los datos de pobreza por parroquias en la ciudad de Quito se recogen en la FIG. 3 para que puedan ser utilizados en investigaciones relacionadas.

En tanto a las variables de movilidad independientes a incluir en el modelo la variable (a_distanciaentiempolñaquito) se calcula como el tiempo que toma llegar desde el centro de cada parroquia hasta el centro de la parroquia de Iñaquito. Para ello se calcula el (a.1 tiempoalcentro) tiempo que tardan los habitantes de cada parroquia en llegar a pie hasta el centro de su parroquia y el (a.2_tiempodelcentroparroquiaalñaquito) tiempo en transporte público desde el centro de cada parroquia hasta el centro de Iñaquito 9 .

\footnotetext{
${ }^{6}$ La parroquia de Iñaquito es la que más empleos genera en Quito, con el $22,5 \%$ del total. Su identificación se realizó en base al análisis de una muestra de 347 empresas de las casi 3.600 empresas activas con sede en la ciudad de Quito en 2010 proporcionada por el Municipio de Quito.

${ }^{7}$ La pérdida de representatividad es lo que ha evitado el uso del Índice de Necesidades Básicas que sí está por parroquia pero que se basa tanto en el Censo como en la ENEMDU.

${ }^{8} \mathrm{Se}$ han unificado las bases del censo de vivienda, hogar,
}

emigración y población para no duplicar registros y considerar únicamente los datos de viviendas con su correspondencia en hogares y población.

9 Los medios de transporte considerados son todos los que se utilizan en Quito, que son la Ecovía, Corredor Central Trolebus, Corredor Central Norte, Corredor Sur oriental, Troncal Suroccidental, Rutas del Sistema Integrado, Ramales, Rutas Interparroquiales e Intraparroquiales y Rutas Convencionales e Individuales. 


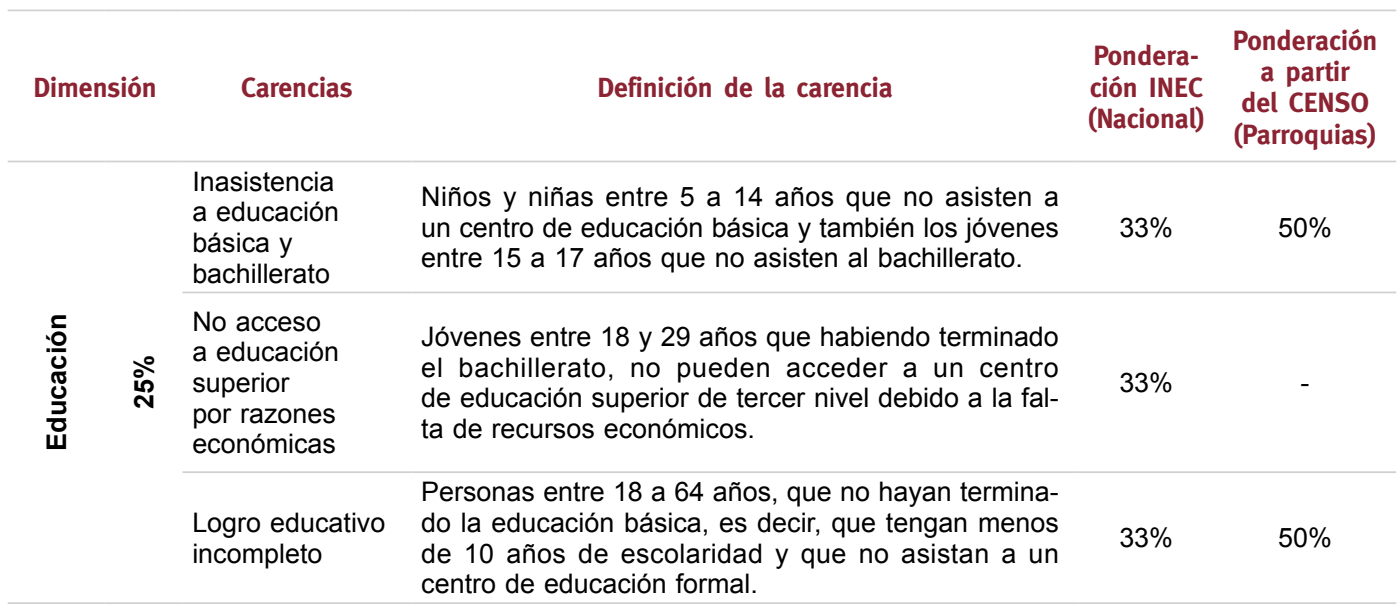

Niños y niñas entre 5 a 14 años que estén ocupados en la semana de referencia se identifican como privados al considerarse prohibido el trabajo infantil. Para

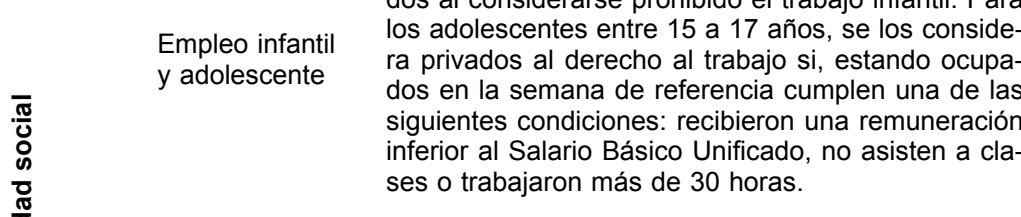

Desempleo

o empleo

inadecuado

Personas de 18 años o más, que, en el período de referencia, estuvieron desocupadas. Adicionalmente, se consideran privadas las personas ocupadas que tienen un empleo inadecuado.

Personas ocupadas de 15 años o más, que no aportan a ningún tipo de seguridad social; excluyendo de la privación a personas ocupadas de 65 años y más,

No contribución al sistema de pensiones que no aportan, pero reciben pensión por jubilación. Para personas en condición de desempleo o económicamente inactivas, de 65 años o más, se las considera en privación si no reciben pensión por jubilación, Bono de Desarrollo Humano o Bono Joaquín Gallegos Lara.

\begin{tabular}{|c|c|c|c|c|c|}
\hline \multirow{2}{*}{ 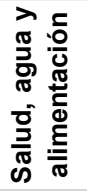 } & \multirow{2}{*}{ ஷั้ } & $\begin{array}{l}\text { Sin servicio } \\
\text { de agua } \\
\text { por red pública }\end{array}$ & $\begin{array}{l}\text { Miembros de las viviendas que obtienen el agua por } \\
\text { un medio distinto al de la red pública. }\end{array}$ & $50 \%$ & $100 \%$ \\
\hline & & $\begin{array}{l}\text { Pobreza extrema } \\
\text { por ingresos }\end{array}$ & $\begin{array}{l}\text { Personas cuyo ingreso per cápita familiar es inferior } \\
\text { al de la línea de pobreza extrema. }\end{array}$ & $50 \%$ & - \\
\hline \multirow{4}{*}{ 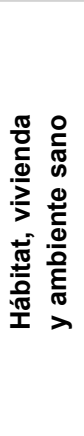 } & \multirow{4}{*}{ ڤั } & Hacinamiento & $\begin{array}{l}\text { Miembros de viviendas que tienen más de tres perso- } \\
\text { nas por dormitorio exclusivo para dormir. }\end{array}$ & $25 \%$ & $25 \%$ \\
\hline & & $\begin{array}{l}\text { Déficit } \\
\text { habitacional }\end{array}$ & $\begin{array}{l}\text { Personas cuya vivienda, debido a los materiales o } \\
\text { estado de sus paredes, piso y techo, son considera- } \\
\text { das en déficit cualitativo o cuantitativo. }\end{array}$ & $25 \%$ & $25 \%$ \\
\hline & & $\begin{array}{l}\text { Sin saneamiento } \\
\text { de excretas }\end{array}$ & $\begin{array}{l}\text { Personas del área urbana cuya vivienda no cuenta } \\
\text { con servicio higiénico conectado a alcantarillado. En } \\
\text { el área rural, las personas privadas son aquellas } \\
\text { cuya vivienda no cuenta con alcantarillado o pozo } \\
\text { séptico. }\end{array}$ & $25 \%$ & $25 \%$ \\
\hline & & $\begin{array}{l}\text { Sin servicio } \\
\text { de recolección } \\
\text { de basura }\end{array}$ & $\begin{array}{l}\text { Personas que habitan en viviendas que no tienen ac- } \\
\text { ceso al servicio municipal de recolección de basura, } \\
\text { se clasifican como privadas en este indicador. }\end{array}$ & $25 \%$ & $25 \%$ \\
\hline
\end{tabular}

IG. 3/ Variables para calcular el IPM por parroquias en Quito y por cantón a nivel nacional. 
El cálculo sigue la siguiente Fórmula (2):

(2) a_distanciaentiempolñaquito $_{i}=$ a.1_tiempoalcentro + a.2_tiempodelcentroparroquiaalñaquito

Para calcular el (a.1_tiempoalcentro) tiempo a pie hasta el centro de la parroquia se mide la distancia en kilómetros hasta el centro de cada parroquia y se multiplica por la velocidad a pie, de $4,66 \mathrm{~km} / \mathrm{h}$, aproximación realizada por la Universidad de Wisconsin (GATES \& al., 2006).

Para calcular el (a.2_tiempodelcentroparroquiaalñaquito), cuánto se tarda desde el centro de cada parroquia hasta el centro de Iñaquito se mide la ruta más probable en kilómetros tomada en transporte público considerando un medio o más, en función del caso, y se multiplica por la velocidad máxima para el transporte público de pasajeros en el sector urbano de $40 \mathrm{~km} / \mathrm{h}$ (Reglamento a la Ley de Transporte Terrestre, Tránsito y SEguridad ViaL, 2012).

La variable independiente (b_densidadtp), que mide la disponibilidad de medios de transporte público por parroquia se calcula multiplicando la (b.1_líneas) cantidad de líneas de transporte en la parroquia por la (b.2_frecuencia) por la frecuencia de paso entre el total de la población por parroquia.

La información fue facilitada por la Secretaría de Movilidad del Municipio de Quito. Las líneas disponibles se han calculado incluyendo todas las que están en funcionamiento ${ }^{10}$, mientras que la frecuencia excluye el número de articulados y biarticulados de la Ecovía y el Corredor Central Trolebús, por ser constante. La (b.2) frecuencia recoge el número de unidades de los medios de transporte considerados disponibles por hora en cada parroquia entre la población de la parroquia. El cálculo sigue la siguiente Fórmula (3):

(2) 『b_densidadtp $\rrbracket \_i=\llbracket b .1$ lineas $\rrbracket \_i$ $+\llbracket b .2$ frecuencia $\rrbracket i$

\section{Resultados}

El modelo indica que, en conjunto, la distancia, la densidad de los medios de transporte y la etnia explican en un $76 \%$ la pobreza para las 31 parroquias estudiadas. Todas las variables resultan significativas. Los signos de los coeficientes son coherentes. Contra más densidad menos pobreza, a más distancia más pobreza y siendo indígena, la probabilidad de ser pobre aumenta (FIG. 4).

Se realizó un conjunto de pruebas de robustez para comprobar la validez del modelo. Para analizar la correcta especificación del modelo, se utilizó el contraste RESET de Ramsey siendo la hipótesis nula la no omisión de variables, la cual no se puede rechazar a un nivel de significancia razonable, por lo que se concluye que combinaciones no lineales de las variables independientes no explicarían la variable dependiente. Con el objetivo de comprobar que la varianza de los términos de perturbación sea constante, se utilizó el test de Breusch-Pagan que arrojó un resultado menor a 0,5 , lo que permite no rechazar la hipótesis nula de que existe homocedasticidad

\begin{tabular}{r|crc} 
Source & SS & df & MS \\
\hline $\begin{array}{r}\text { Model } \\
\text { Residual }\end{array}$ & .030551912 & 3 & .010183971 \\
.009165192 & 27 & .000339452 \\
\hline Total & .039717104 & 30 & .001323903
\end{tabular}

$\begin{array}{llr}\text { Number of obs } & = & 31 \\ \text { F (3, 27) } & =30.00 \\ \text { Prob }>\text { F } & =0.0000 \\ \text { R-squared } & =0.7692 \\ \text { Adj R-squared } & =0.7436 \\ \text { Root MSE } & =.01842\end{array}$

\begin{tabular}{r|rrrrrr}
\hline ipm & Coef. & Std. Err. & $t$ & P > t l & [95\% Conf. Interval] \\
\hline densidadpob & -.0125612 & .0053381 & -2.35 & 0.026 & -.023514 & -.0016084 \\
distancia & .0006776 & .0003923 & 1.73 & 0.096 & -.0001274 & .0014826 \\
indigena & .0000149 & $2.26 e-06$ & 6.59 & 0.000 & .0000103 & .0000195 \\
_cons & .0286726 & .0098365 & 2.91 & 0.007 & .0084897 & .0488555
\end{tabular}

Resultados del modelo de correlación de exclusión por movilidad. 
a cualquier nivel de significancia razonable. Por otro lado, para comprobar el supuesto de que las variables explicativas deben ser linealmente independientes se utilizó la prueba VIF (Variance Inflation Factor), misma que resulta menor a cinco por lo que se concluye que no existe multicolinealidad. Finalmente, para testear la distribución normal de los errores se hizo uso de la prueba de Shapiro-Wilk, con un resultado menor a uno. A un nivel de significancia razonable esta prueba reflejó que no se puede rechazar la hipótesis nula, denotando que los errores si se encuentran distribuidos normalmente. En la FIG. 5 se recoge el análisis de las pruebas.

Ramsey RESET test using powers of the fitted values of ipm

$$
\begin{gathered}
F(3,19)=0.83 \\
\text { Prob }>F=0.4956
\end{gathered}
$$

Breusch-Pagan / Cook-Weisberg test for heteroskedasticity

Ho: Constant variance

Variables: residuals

$$
\text { chi2(1) }=0.87
$$

Prob $>$ chibar2 $=0.3506$

\begin{tabular}{|ccc|}
\hline Variable & VIF & 1 NIF \\
\hline indigena & 1.98 & 0.504936 \\
\hline distancia & 1.59 & 0.628674 \\
\hline densidadpob & 1.32 & 0.760382 \\
\hline Mean VIF & 1.63 & \\
\hline
\end{tabular}

Shapiro - Wilk W test for normal data

$\begin{array}{cccccc}\text { Variable } & \text { Obs } & \text { W } & \text { V } & z & \text { Prob }>\text { z } \\ \text { residuals } & 31 & 0.97392 & 0.746 & -0.601 & 0.72624\end{array}$

Ramsey RESET test using powers of the fitted values of ipm

$F(3,19)=0.54$

Prob $>F=0.6603$

Breusch-Pagan / Cook-Weisberg test for heteroskedasticity

Ho: Constant variance

Variables: residuals $\operatorname{chi} 2(1)=0.54$

Prob > chibar2 $=0.4604$

\begin{tabular}{|ccc}
\hline Variable & VIF & 1/VIF \\
\hline indigena & 1.30 & 0.772026 \\
\hline distancia & 1.25 & 0.798374 \\
\hline densidadpob & 1.08 & 0.928213 \\
\hline Mean VIF & 1.21 & \\
\hline
\end{tabular}

Shapiro - Wilk W test for normal data

$\begin{array}{cccccc}\text { Variable } & \text { Obs } & \text { W } & \text { V } & \text { z } & \text { Prob > z } \\ \text { residuals } & 31 & 0.95559 & 1.447 & 0.765 & 0.22217\end{array}$

FIG. 5/ Resultados de pruebas de robustez del modelo de correlación de exclusión por movilidad.

Fuente: Elaboración propia 


\section{Conclusiones}

El análisis econométrico con todas las parroquias urbanas excluyendo las parroquias del centro muestra cómo la distancia en tiempo hasta la principal fuente de ingresos y la densidad de los medios de transporte en cada parroquia contribuyen a generar pobreza. Es necesario entonces plantear dos posibles estrategias. Una es mejorar la movilidad para llegar al puesto de trabajo en menos tiempo y con más medios disponibles mejorando la frecuencia del transporte, incluyendo nuevos medios, como el metro o los funiculares, e impulsando campañas que desestimen el uso del vehículo privado, a favor del público. La otra estrategia sería descentralizar en la medida de lo posible la actividad económica de la ciudad impulsando la capacidad de las centralidades para generar más y mejor empleo. Cualquiera de las dos estrategias necesita implementarse de cara a minimizar la exclusión por movilidad laboral, y su relación positiva sobre la pobreza multidimensional en la ciudad de Quito.

\section{Bibliografía}

Abrahamson, P. (1997): Exclusión Social en Europa: ¿Vino Viejo en Odres Nuevos? En L. Moreno, Unión Europea y Estado del Bienestar (pp. 117-141). Madrid: CSIC.

Alesina, A. \& al. (1999): Budget institutions and fiscal performance in Latin America. Journal of Development Economics, 59(2), 253-273.

https://doi.org/10.1016/S0304-3878(99)00012-7

Alkire, S. \& Foster, J. (2007): Counting and multidimensional poverty measures. (OPHI Working Paper No. 7). University of Oxford.

Arriba, A. (2002): El concepto de exclusión en política social. Unidad de Políticas Comparadas. Recuperado de

http://digital.csic.es/bitstream/10261/1495/1/ dt-0201.pdf

Banco Mundial (2019): Base de datos. Indicadores de desarrollo mundial. Washington: Banco Mundial.

Baradaran, S. \& Ramjerdi, F. (2001): Performance of accessibility measures in Europe. Journal of Transportation and Statistics, 4(2/3), 31-48.

BARRIGA, S. (2005): El trabajo como vía prioritaria contra la exclusión social. En Tendencias en exclusión social y políticas de solidaridad. Octavo foro sobre tendencias sociales. Madrid: Sistema.
BAUer, P. T. (1957): Economic Analysis and Policy in Underdeveloped Countries. Durham: Duke University Press.

Beckmann, M. J. \& Golob, T. F. \& Zahavi, Y. (1983): Travel probability fields and urban spatial structure: 2. Empirical tests. Environment and Planning A: Economy and Space, 15(6), 727-738.

https://doi.org/10.1068/a150727

Воотн, C. (1899): Old Age Pensions and the Aged Poor. London: Macmillan and Co.

Bustamante-Patiño, B., \& Herrero-Olarte, S. (2017): La clase dominante como determinante de la forma de Quito. Bitácora Urbano Territorial, 27(3), 81-90.

https://doi.org/10.15446/bitacora.v27n3.55932

CARRIÓn, D. \& al. (1987): La renta del suelo y segregación urbana en Quito. En F. Carrión \& al. (Eds.), El proceso urbano en el Ecuador (pp. 81-122). Quito: ILDIS.

CARRIÓn, F. (Ed.). (1989): La investigación urbana en América Latina. Caminos recorridos y por recorrer. Estudios nacionales. Quito: CIUDAD.

CAss, N. \& Shove, E. \& URRY, J. (2005): Social exclusion, mobility and access. The Sociological Review, 53(3), 539-555. https://doi.org/10.1111/j.1467-954X.2005.00565.x

Church, A. \& Frost, M. \& Sullivan, K. (2000): Transport and social exclusion in London. Transport Policy, 7(3), 195-205. https://doi.org/10.1016/S0967-070X(00)00024-X

Comisión de las Comunidades EuRopeAs (1992): Transport Hacia una Europa de la Solidaridad. Intensificación de la lucha contra la exclusión social y la promoción de la integración., 7 Bruselas: COM.

- (1993): Libro blanco. La política social europea. Un paso adelante para la Unión. Luxemburgo: Oficina de Publicaciones Oficiales de las Comunidades Europeas.

- (1994): Libro verde. Política social europea. Opciones para la Unión. Luxemburgo: Oficina de Publicaciones Oficiales de las Comunidades Europeas.

DAvis, J. (1959): A formal interpretation of the Theory of the relative Deprivation. Sociometry, 22(4), 280-296.

DE HAAN, A. (1999): Social Exclusion: Towards an holistic understanding of deprivation. Brighton: Institute of Development Studies, University of Sussex.

Deleeck, H. \& Van Den Bosch, K. (1992): Poverty and Adequacy of Social Security in Europe: A Comparative Analysis. Journal of European Social Policy, 2(2), 107-120. https://doi.org/10.1177/095892879200200203 
Demery, D. \& Demery, L. (1973): Cross-Section Evidence for Balanced and Unbalanced Growth. The Review of Economic and Statistics, 55(4), 459-464.

Desal, M. (2001): Amartya Sen's contribution to development economics. Oxford Development Studies, 29(3), 213-223. https://doi.org/10.1080/13600810120088831

DREWNowskI, J. \& ScotT, W. (1966): The Level of Living Index. Geneva: United Nations Research Institute for Social Development.

Drèze, J. \& Sen, A. (1991): The Political Economy of Hunger: Entitlement and Well-being (Volume 1). Oxford: Clarendon Press Oxford.

DURÁN, G. \& MARTI, M. \& MÉRIDA, J. (2016): Crecimiento, segregación y mecanismos de desplazamiento en el periurbano de Quito. Íconos, 56, 123-146. https://doi.org/10.17141/iconos.56.2016.2150

FERIA TORIBIO, J. M. (2010): La delimitación y organización espacial de las areas metropolitanas españolas: una perspectiva desde la movilidad residencia-trabajo. Ciudad y Territorio Estudios Territoriales (CyTET), 42(164),189 210. Recuperado a partir de https://recyt.fecyt.es/index.php/CyTET/article/ view/75989

Fromm, E. (1941): Escape from Freedom. New York: Henry Holt and Companym LLC.

GATES, T. \& al. (2006): Recommended Walking Speeds for Pedestrian Clearance Timing Based on Pedestrian Characteristics. Recuperado de https://pdfs.semanticscholar.org/fc81/aff9a47546a8034cf0d94438ed7466c97326.pdf

Golledge, R. \& Stimson, R. (1997): Spatial Behaviour: A Geographic Perspective. New York.

Harrington, M. (1984): The New American Poverty. New York: Holt Rinehart \& Winston.

Heilbroner, R. (1976): Business Civilization in Decline. New York: W. W. Norton \& Company.

HernÁndez, M. (2008): Exclusión social y desigualdad. (Vol. 6). EDITUM

Herrero-Olarte \&, S., Villa, A. \& Sandoval, S. (2019): ¿Influye la diversidad económica del barrio en su tasa de pobreza? El caso de Quito. ACE: Architecture, City and Environment, 13(39), 145-160.

Hicks, N. L. (1979): Growth vs basic needs: Is there a trade-off?. World Development, 7(11), 985-994. https://doi.org/10.1016/0305-750X(79)90066-4

Hicks, N. L. y Streeten, P. (1979): Indicators of development: The search for a basic need's yardstick. World Development, 7(6), 567-580. https://doi.org/10.1016/0305-750X(79)90093-7

Instituto Nacional de Estadísticas y Censos. INEC. (2011): Censo de Población y Vivienda 2010.
Recuperado de

http://www.ecuadorencifras.gob.ec/base-de-datos-censo-de-poblacion-y-vivienda-2010/

- (2016): Índice de Pobreza Multidimensional. Recuperado de

https://www.ecuadorencifras.gob.ec/pobrezamultidimensional/

JAMESON, K. P. (1980): Supply Side Economics: Growth versus Income Distribution. Challenge, 23(5), 26-31.

Jolly, R. (2008): A Short History of IDS: A Personal Reflection. (IDS Discussion Paper 388). Institute of Development Studies

KABEER, N. (2010): Can the MDGs provide a pathway to social justice? The challenge of intersecting inequalities. New York: Institute of Development Studies.

Leal Maldonado, J. \& Domínguez Pérez, M. (2008): "Transformaciones económicas y segregación social en Madrid". Ciudad Y Territorio Estudios Territoriales (CyTET), 40(158), 703-725. Recuperado a partir de

https://recyt.fecyt.es/index.php/CyTET/article/ view/75895

LenoIR, R. (1974): Les Exclus: Un Francais sur dix. Paris. Editions Le Seuil.

Malthus, T. (1846): Ensayo sobre el principio de la población. Madrid: Establecimiento Literario y Tipográfico de D. Lucas Gonzalez y Compañía.

MARCuSE, H. (1964): El hombre unidimensional. Barcelona: Ariel.

MAsLow, A. (1943): A theory of human motivation. Psychological Review, 50(4), 370-396.

Mawdsley, E. \& Rigg, J. (2003): The World Development Report II: continuity and change in development orthodoxies. Progress in Development Studies, 3(4), 271-286. https://doi.org/10.1191/1464993403ps065oa

MAY, J. \& THRIFT, N. (2001): Timespace: geographies of temporality. Routledge.

McNamara, R. (1972): Discurso ante la Conferencia de las Naciones Unidas sobre Comercio y Desarrollo. Santiago de Chile. Recuperado de http://www-wds.worldbank.org/external/default/ WDSContentServer/WDSP/IB/2011/11/24/0003 56161_20111124004511/Rendered/PDF/42030 OWPOSPANIOnitedONationsSpanish.pdf

Miguel, E. \& Gugerty, M. K. (2005): Ethnic diversity, social sanctions, and public goods in Kenya. Journal of Public Economics, 89(11), 2325-2368. https://doi.org/10.1016/j.jpubeco.2004.09.004

MoRAWETZ, D. (1977): Twenty-five years of economic development. Finance and Development, 14 (3), 10.

MoRAWETZ, H. (1979): Some applications of fluorimetry to synthetic polymer studies. Science, 203(4379), 405-410. 
NNUU (2014): World urbanization prospects: The 2014 revision. New York: United Nations Department of Economics and Social Affairs, Population Division

Nurkse, R. (1953): Problems of Capital Formation in Underdeveloped Countries. New York: Oxford University Press.

Parrado-Rodríguez, C. (2018): Rehabilitación urbana y pobreza oculta. La Mariscal, Quito. Bitácora Urbano Territorial, 28(2), 17-24. https://doi.org/10.15446/bitacora.v28n2.70065

Pearson, L. B. (1969): Partners in Development: Report of the Commission of International Development. New York: Praeger.

PREBISCH, R. (1950): The Economic Development of Latin America and its Principal Problems. New York: ONU.

RAYA, E. (2005): Categorías sociales y personas en situación de exclusión. Una aproximación desde el País Vasco. Cuadernos de Relaciones Laborales, 23(2), 247-267.

Reglamento a la Ley de Transporte Terrestre, Tránsito y Seguridad Vial. (2012): Registro Oficial Suplemento 731 de 25 de junio de 2012.

RoBERTs, E. (1977): Working-Class Standards of Living in Barrow and Lancaster, 1890-1914. The Economic History Review, 30(2), 306-321. https://doi.org/10.1111/j.1468-0289.1977. tb00267.x

Rosenstein-Rodan, P. N. (1943): Problems of Industrialization in Eastern and Southeastern Europe. The Economic Journal, 53(210/211), 202-211.

https://doi.org/10.2307/2226317

SAITH, R. (2001): Social Exclusion: The Concept and Application to Developing Countries. Oxford. (QEH Working Paper Series QEHWPS72). University of Oxford. Recuperado de

http://workingpapers.qeh.ox.ac.uk/RePEc/qeh/ qehwps/qehwps72.pdf

Schönfelder, S. \& Axhausen, K. W. (2002): Measuring the size and structure of human activity spaces-The longitudinal perspective. Arbeitsbericht Verkehrs- und Raumplanung, $135,1-47$.

https://doi.org/10.3929/ethz-a-004444846

Scitovsky, T. (1954): Two Concepts of External Economies. Journal of Political Economy, 62(2), 143-151.

SEERS, D. (1969): The meaning of development. (IDS Communication, 44). Institute of Development Studies. Recuperado de

Sen, A. (1981): Poverty and Famines: An Essay on Entitlement and Deprivation. New York: Oxford University Press.
- (1982): Utilitarianism and Beyond. Cambridge: Cambridge University Press.

- (1984). Resources, Values and Development. Oxford: Basil Blackwell.

- (1999). Development as Freedom. Oxford. Oxford University Press.

- (2003). La libertad individual como compromiso social. Plural editores.

- (2008): The Idea of Justice. Journal of Human Development, 9(3), 331-342. https://doi.org/10.1080/14649880802236540

Shashua, L. \& Goldschmidt, Y. (1972): The Merits of Balanced Growth Reconsidered. The Review of Economics and Statistics, 54(3), 337-339.

SHove, E. (2002): Rushing around: coordination, mobility and inequality. London: Department of Sociology, Lancaster University.

SILVER, H. (1994): Exclusión social y solidaridad: Tres paradigmas. Revista Internacional del Trabajo, 113(5-6), 607-662.

Sraffa, P. (1986): On the Principles of Political Economy and Taxation. Cambridge: Cambridge University Press.

Stouffer, S. A. \& al. (1949): The American soldier: Adjustment during army life. New Jersey: Princeton University Press.

StREETEN, P. (1984): Basic needs: some unsettled questions. World Development, 12(9), 973-978. https://doi.org/10.1016/0305-750X(84)90054-8

Streeten, P. \& al (1981): First Things First: Meeting Basic Human Needs in Developing Countries. New York: Oxford University Press.

Swamy, D. S. (1967): Statistical Evidence of Balanced and Unbalanced Growth. The Review of Economic and Statistics, 49(3), 288-303.

Tezanos, J. F. (1999): Tendencias en desigualdad y exclusión social: Tercer foro sobre Tendencias Sociales. Madrid: Sistema.

Von Hoegen, M. \& Palma, D. (2000): Los pobres explican la pobreza: el caso de Guatemala. Guatemala: Universidad Rafael Landivar.

WiLson, W. J. (1987): The Truly Disadvantaged. The Inner City, the Underclass and Public Policy. Chicago: Chicago University Press.

\section{Lista de acrónimos o siglas}

\begin{tabular}{|c|c|}
\hline CEPAL: & $\begin{array}{l}\text { Comisión Económica para América } \\
\text { Latina }\end{array}$ \\
\hline EEUU: & Estados Unidos \\
\hline ENEMDU: & $\begin{array}{l}\text { Encuesta Nacional de Empleo, } \\
\text { Desempleo y Subempleo }\end{array}$ \\
\hline ENSANUT: & $\begin{array}{l}\text { Encuesta Nacional de Salud } \\
\text { y Nutrición }\end{array}$ \\
\hline
\end{tabular}


IDS:

INEC:

Institute of Development Studies Instituto Nacional de Estadísticas y Censos

IP:

$\mathrm{IPH}:$

IPM:

MCO:

$\mathrm{NBI}$ :
Intensidad de la Pobreza

Índice de Pobreza Humana

Índice de Pobreza Multidimensional

Mínimos Cuadrados Ordinarios

Necesidades Básicas Insatisfechas
NNUU: $\quad$ Naciones Unidas

OPHI: Oxford Poverty \& Human

Development Initiative

PIB: $\quad$ Producto Interno Bruto

PNB: $\quad$ Producto Nacional Bruto

TPM: $\quad$ Tasa de Pobreza Multidimensional

UNRISD: United Nations Research Institute

for Social Development 